\title{
Solidão em idosos e fatores associados
}

\author{
Loneliness in the elderly and associated factors
}

Soledad en ancianos y factores asociados

Recebido: $24 / 08 / 2020$

Aprovado: 28/12/2020

Publicado: 27/01/2021

\author{
Heloísa Gonçalves Ferreira1 \\ Níldila Villa Casemiro²
}

Este é um estudo quantitativo, de corte transversal do tipo descritivo e correlacional. A coleta de dados ocorreu entre agosto de 2017 e abril de 2019, na cidade de Uberaba, com o objetivo de analisar a frequência da solidão em idosos e relações entre solidão com prática de atividades prazerosas, depressão, bem-estar subjetivo e autoavaliação de saúde. Foram aplicados instrumentos de avaliação da solidão, atividades prazerosas, bem-estar subjetivo e depressão, além de um questionário sociodemográfico em idosos não institucionalizados e sem comprometimento cognitivo. Foram pesquisados 156 idosos com idades entre 60 e 88 anos (M=69,72; $\mathrm{DP}=6,77)$, sendo 83,3\% do sexo feminino e $16,7 \%$ do sexo masculino. Um total de $10,9 \%$ apresentou solidão moderada a grave. Foram encontradas relações significativas entre solidão e os demais construtos: quanto maior o nível de solidão, mais sintomas de depressão, pior a autoavaliação em saúde e menor a prática de atividades prazerosas e bem-estar. Os resultados apontam para prováveis fatores de risco e de proteção para solidão em idosos, e por isso apresentam implicações relevantes para o planejamento de intervenções e políticas públicas que visem aprimorar a saúde mental desse público.

Descritores: Envelhecimento; Saúde mental; Promoção da saúde; Prazer.

This is a quantitative, cross-sectional study of a descriptive and correlational type. Data collection took place between August of 2017 and April of 2019, in the city of Uberaba, MG, Brazil. It aimed to analise the frequency of loneliness in the elderly and relationships between loneliness with the practice of pleasurable activities, depression, subjective well-being and self-rated health. Instruments for assessing loneliness, pleasurable activities, subjective well-being and depression were applied, in addition to a sociodemographic questionnaire in non-institutionalized elderly people without cognitive impairment. 156 elderly people aged between 60 and 88 years were surveyed $(M=69.72$; SD $=6.77), 83.3 \%$ being female and $16.7 \%$ male. A total of $10.9 \%$ had moderate to severe loneliness. Significant relationships were found between loneliness and the other constructs: the higher the level of loneliness, the more symptoms of depression, the worse the self-rated health and the lesser the practice of pleasurable activities and well-being. The results point to probable risk and protective factors for loneliness in the elderly, and therefore have relevant implications for planning interventions and public policies aimed at improving the mental health of this public.

Descriptors: Aging; Mental health; Health promotion; Pleasure.

Descriptores: Envejecimiento; Salud mental; Promoción de la salud; Placer.

Este es un estudio cuantitativo y transversal de tipo descriptivo y correlativo. La recogida de datos tuvo lugar entre agosto de 2017 y abril de 2019, en la ciudad de Uberaba, MG, Brasil, con el objetivo de analizar la frecuencia de la soledad en ancianos y las relaciones entre la soledad y la práctica de actividades placenteras, la depresión, el bienestar subjetivo y la autoevaluación de la salud. Se aplicaron instrumentos de evaluación de la soledad, actividades placenteras, bienestar subjetivo y depresión, además de un cuestionario sociodemográfico en ancianos no institucionalizados y sin deterioro cognitivo. Se encuestó a un total de 156 ancianos de entre 60 y 88 años (M=69,72; SD=6,77), 83,3\% mujeres y $16,7 \%$ hombres. Un total del 10,9\% presentó soledad moderada a severa. Se encontraron relaciones significativas entre soledad y otros constructos: cuanto más alto es el nivel de soledad, más síntomas de depresión, peor es la autoevaluación de la salud y menor es la práctica de actividades placenteras y de bienestar. Los resultados apuntan a probables factores de riesgo y de protección de la soledad en ancianos y, por lo tanto, tienen implicaciones relevantes para la planificación de intervenciones y políticas públicas destinadas a mejorar la salud mental de este público.

Descriptores: Envejecimiento; Salud mental; Promoción de la salud; Placer. 


\section{INTRODUÇÃO}

A

solidão pode ser compreendida como a percepção do indivíduo sobre o quanto seus relacionamentos interpessoais são satisfatórios, tanto em termos de quantidade como em qualidade ${ }^{1}$. Não se trata apenas de estar ou não na presença de outras pessoas, pois o indivíduo pode estar em contato com várias delas e ainda assim relatar sentir-se só ${ }^{1,2}$. Ou seja, a solidão diz sobre uma falta de conexão social experimentada de forma subjetiva pelo indivíduo, que inclui componentes emocionais e cognitivos que exprimem percepções e sensações de isolamento e vazio nas relações ${ }^{3}$.

Atualmente a solidão representa um problema de caráter epidêmico, sendo frequente em idosos ${ }^{4}$. Nesta população, especificamente, estudos internacionais demonstram que a solidão aparece associada à depressão, ao isolamento social e a morar sozinho ${ }^{5}$. Queixas frequentes de solidão em idosos também estão associadas a eventos normativos de transição ao longo da vida, tais como a saída dos filhos de casa, viuvez e aposentadoria ${ }^{6}$. A solidão em idosos pode estar ainda relacionada a um déficit no suporte social, à perda de autonomia e deterioração da saúde e consequente diminuição do bem-estar ${ }^{7}$.

Logo, a solidão traz implicações importantes para a saúde física e mental das pessoas, sendo relevante a elucidação de possíveis fatores de risco e de proteção associados à manifestação da solidão. Considerando os possíveis fatores de proteção para solidão em idosos, a promoção de contatos sociais na terceira idade por meio de grupos de convivência pode proporcionar bem-estar subjetivo ${ }^{8}$ e ajudar a amenizar sentimentos de solidão em idosos ${ }^{9}$. Portanto, contextos na qual idosos têm oportunidades de estabelecer relações interpessoais significativas e praticar atividades prazerosas parecem ser protetivos à saúde mental, podendo resultar em menores níveis de solidão em idosos ${ }^{10}$.

Ainda, os estudos que investigam a solidão em idosos no contexto brasileiro são incipientes, havendo poucos dados sobre como a solidão se manifesta e com quais fatores apresenta relação para esse público em específico. No Brasil, as pessoas com 60 anos ou mais representam cerca de $13 \%$ da população. Esse número, entretanto, tende a dobrar nas próximas décadas ${ }^{11}$.

Considerando-se o crescimento acelerado da população idosa brasileira, entende-se que investigações sobre a saúde mental de idosos são imprescindíveis para a elaboração e implementação de intervenções e políticas públicas para melhorar as condições de envelhecimento da população no Brasil. Desta forma, é importante a realização de estudos que investiguem as relações entre solidão com aspectos da saúde mental de idosos, visando identificar possíveis fatores de risco e de proteção para a vivência da solidão. Desse modo, o presente estudo tem por objetivo investigar a frequência da solidão em idosos e relações entre solidão com prática de atividades prazerosas, depressão, bem-estar subjetivo e autoavaliação de saúde.

\section{MÉTODO}

Este é um estudo quantitativo, de corte transversal do tipo descritivo e correlacional. A coleta de dados ocorreu entre agosto de 2017 e abril de 2019, na cidade de Uberaba. 0 presente estudo foi derivado do projeto de pesquisa intitulado: Indicadores de saúde mental em idosos de Uberaba: um estudo descritivo, o qual foi encaminhado e aprovado pelo Comitê de Ética em Pesquisa da Universidade Federal do Triângulo Mineiro (CAAE: 65813417.9.0000.5154).

Para este estudo considerou-se amostra por conveniência. Foram adotados os seguintes critérios de inclusão: pessoas com mais de 60 anos, não institucionalizadas e frequentadoras dos locais de recrutamento selecionados para este estudo (grupos de convivência para idosos, Serviço-Escola em Psicologia, grupo Hiperdia do Hospital das Clínicas da Universidade Federal do Triângulo Mineiro - HC/UFTM). 0 critério de exclusão consistiu em idosos que apresentassem comprometimento cognitivo referenciado pelos profissionais que 
acompanhavam esses idosos nos centros de recrutamento. Quando recrutados no Hospital das Clínicas e em Serviço Escola de Psicologia, o comprometimento cognitivo foi aferido pelo MiniExame do Estado Mental, sendo considerados para o estudo apenas os idosos que atingissem a pontuação de corte indicativa de ausência de comprometimento cognitivo12.

Um questionário sociodemográfico foi utilizado para obter informações de caracterização dos idosos, incluindo a autoavaliação da saúde, que consistiu na seguinte pergunta: Em geral você diria que a sua saúde é? 1 - excelente; 2 - muito boa; 3 - boa; 4 - razoável; 5 - ruim.

Foi aplicada a Escala Brasileira de Solidão (UCLA-BR). 0 instrumento é uma escala para adultos e idosos que foi adaptada para o Brasil $^{3}$, com base nas versões estrangeiras UCLA-R ${ }^{13} \mathrm{e}$ UCLA- $3^{14}$. A UCLA-BR é composta por 20 afirmações que retratam os estados afetivos e cognitivos da solidão. 0 participante assinala a frequência, que pode variar de 1 (nunca) a 4 (frequentemente), com que experimentou ultimamente as situações descritas nos itens. A escala apresentou consistência interna satisfatória $(\alpha=0,94)$ e apresentou evidências de validade externa, visto que possui relações significativas com depressão e suporte social. A pontuação de corte para interpretação são: 0 a 22 pontos, indicativo de solidão mínima; 23 a 35 pontos, indicativo de solidão leve; 36 a 47 pontos, indicativo de solidão moderada; e 48 a 60 pontos, indicativo de solidão intensa ${ }^{15}$.

Para avaliar a PAP (Práticas de Atividades Prazerozas), foi utilizada a Versão brasileira adaptada do California Older Person's Pleasant Events Schedule ${ }^{16}$, nomeadamente o OPPES-BR ${ }^{17}$. A escala aplicada é composta por 67 itens que descrevem atividades que tendem a ser agradáveis para idosos. 0 participante assinala a frequência que realizou tais atividades no último mês conforme a escala de pontuação: 0 (nunca); 1 (1 - 6 vezes), e 2 (mais de 7 vezes). Além disso, ele deve classificar o prazer subjetivo experienciado em cada atividade que realizou ou caso tivesse realizado, utilizando a escala de pontuação: 0 (não foi ou não teria sido agradável), 1 (foi ou teria sido razoavelmente agradável) e 2 (foi ou teria sido bastante agradável).

0 PAP possui uma estrutura de quatro fatores referentes à subescala Frequência18: (1) Atividades Sociais e de Competência $(\alpha=0,94)$ : abrangem situações sociais nas quais o idoso pode expressar um senso de competência e utilidade para si e para os outros; (2) Atividades Contemplativas $(\alpha=0,83)$ : atividades introspectivas em que o idoso pode expressar sentimentos positivos e também estar em contato com a natureza; (3) Atividades Práticas $(\alpha=0,72)$ : eventos presentes na rotina do idoso, como atividades domésticas e de envolvimento com a comunidade; e (4) Atividades Intelectuais $(\alpha=0,70)$ : exigem habilidades de ler e escrever para que o idoso as pratique.

Para acessar o bem-estar subjetivo foi utilizada a Escala de Afetos Positivos e Negativos EAPN ${ }^{19}$ e a Escala de Satisfação com a Vida - ESV 20 , as quais foram traduzidas para o Brasil ${ }^{21} \mathrm{e}$ adaptadas para o uso com idosos brasileiros ${ }^{22}$. A EAPN investiga os afetos que as pessoas vivenciam e a ESV possibilita uma avaliação mais global da satisfação pessoal do indivíduo com a relação à vida. A EAPN é composta de dez diferentes afetos (positivos e negativos), sendo que o entrevistado deve responder numa escala de 1 (nada) a 10 (extremamente), o quanto tem experimentado cada afeto ultimamente. Já a ESV é composta por 4 afirmações que o entrevistado deve responder numa escala de 1 (discordo totalmente) a 10 (concordo plenamente), com relação a sua satisfação com a vida. Ambas as escalas apresentam boas consistências internas ( $\alpha=0,78$ para a EAPN e $\alpha=0,84$ para a ESV).

Foi aplicada a Escala de Depressão Geriática - versão reduzida - (EDG-15). A EDG-15 foi desenvolvida ${ }^{23}$ e traduzida e validada para o Brasil24. É composta por 15 itens, sendo um dos instrumentos mais usados para detecção de sintomas depressivos graves e leves em idosos, em pesquisas e na prática clínica ${ }^{23}$. Pontuações acima de 5 indicam triagem positiva para depressão. Apresenta especificidade de 73,9\% e sensibilidade de 85,4\%.

Para a coleta de dados, os idosos foram contactados em grupos de convivência (Unidade de Atenção ao Idoso e Universidades Abertas à Terceira Idade), grupo Hiperdia do HC/UFTM e 
Serviço Escola em Psicologia vinculados à Instituição do Comitê de Ética onde a pesquisa foi aprovada. Primeiramente, foram consultados os profissionais que atuavam diretamente com os idosos nesses locais, para solicitar indicações de participantes em potencial para a pesquisa.

A partir destas indicações, os idosos eram convidados a participar da pesquisa. Quando o idoso aceitava participar, era combinado previamente a forma de aplicação dos instrumentos, que ocorreu em formato autoadministrado ou por meio de entrevista, a depender das condições do idoso. Graduandos de Psicologia, previamente treinados, realizaram a coleta de dados por meio da aplicação dos instrumentos nas instituições onde o idoso havia sido contatado. Primeiramente era lido o Termo de Consentimento Livre e Esclarecido. Após a assinatura do termo, os instrumentos eram aplicados.

A análise dos dados foi realizada no programa SPSS versão 22. Foram adotadas técnicas de estatística descritiva para investigar médias, desvios padrões, proporções e mínimos e máximos das variáveis, além do Teste de Kolmogorov-Smirnov para investigar a distribuição das variáveis, tendo sido constatado que apresentavam distribuição não-normal. Assim, adotou-se o coeficiente de correlação de Spearman para testar as relações entre os construtos.

\section{RESULTADOS}

Foram pesquisados 156 idosos com idades entre 60 e 88 anos $(M=69,72$; DP=6,77), sendo $83,3 \%$ do sexo feminino e $16,7 \%$ do sexo masculino. A Tabela 1 mostra a frequência de solidão, sendo possível observar que a maior parte dos participantes $(89,1 \%)$ apresentou solidão mínima a leve.

Tabela 1. Níveis de solidão nos idosos (n=156). Uberaba, Agosto de 2017 a Abril de 2019.

\begin{tabular}{crr}
\hline Níveis de Solidão & \multicolumn{1}{c}{$\mathrm{N}$} & \multicolumn{1}{c}{$\%$} \\
\hline Solidão mínima & 114 & $73,1 \%$ \\
Solidão leve & 25 & $16,0 \%$ \\
Solidão moderada & 10 & $6,4 \%$ \\
Solidão intensa & 7 & $4,5 \%$ \\
\hline
\end{tabular}

A Tabela 2 apresenta os valores do coeficiente de correlação para as relações entre solidão e depressão, PAP, BES e AS. Nota-se que solidão apresentou correlações estatisticamente significativas com todos os construtos. Solidão apresentou relações fracas e moderadas com PAP, relação forte com depressão, relação moderada com BES e relação moderada com AS. Deste modo, os resultados indicam que quanto mais solidão o idoso experimenta, menos atividades prazerosas ele pratica, menos BES ele experimenta, mais sintomas de depressão ele sofre e pior ele avalia sua saúde.

Tabela 2. Correlações entre solidão e prática de atividades prazerosas, depressão, bem-estar subjetivo e autoavaliação da saúde. Uberaba, Agosto de 2017 a Abril de 2019.

\begin{tabular}{llc}
\hline & & Solidão \\
\hline & Frequência Geral & $-0.37^{* *}$ \\
Prática de Atividades Prazerosas & Ativer Geral & $-0.20^{* *}$ \\
& Atividades Sociais e de Competência & $-0.38^{* *}$ \\
& Atividades Contemplativas & $-0.25^{* *}$ \\
& Atividades Práticas & $-0.16^{*}$ \\
& Atividades Intelectuais & $-0.19^{*}$ \\
& Afetos Positivos & $-0.50^{* *}$ \\
Bem Estar Subjetivo & Afetos Negativos & $0.51^{* *}$ \\
Depressão & Satisfação com a Vida & $-0.47^{* *}$ \\
Autoavaliação da Saúde & & $0.61^{* *}$ \\
Nota: ${ }^{*}<<.05 ;{ }^{* *}<.01$ & & $0.40^{* *}$ \\
\hline
\end{tabular}




\section{DISCUSSÃo}

Foi constatado que a maioria dos pesquisados apresentou nível mínimo a leve de solidão. Porém, 10,9\% dos idosos apresentou triagem indicativa para solidão moderada a grave. Quando comparado este resultado com estudos que utilizaram versões da UCLA para aferir solidão em idosos de outros países, a proporção de idosos do presente estudo que sofre de níveis mais severos de solidão é menor do que em idosos chineses residentes em zonas rurais, onde $78,1 \%$ apresentou solidão moderada a grave ${ }^{25}$, e em idosos indianos da comunidade, onde $37,6 \%$ apresentou pontuações acima de 50 na UCLA ${ }^{26}$.

Esses resultados sugerem que a solidão em idosos pode ser influenciada também por fatores culturais, uma vez que são observadas proporções discrepantes na prevalência de níveis mais severos de solidão, mensurados a partir de versões do mesmo instrumento em diferentes culturas. No entanto, também é necessário considerar que parte dos idosos do presente estudo foram recrutados em centros de convivência, locais favorecedores da saúde mental de idosos 8 e que, portanto, poderia explicar, ainda que parcialmente, a menor proporção de idosos com triagem positiva para níveis mais severos de solidão.

Ao avaliar as relações encontradas entre solidão com os demais construtos (depressão, PAP, BES e AS), a relação de magnitude mais forte encontrada foi entre solidão e depressão, uma relação já bem documentada para a população geral, seja no contexto nacional ${ }^{2,3}$ quanto em outros países ${ }^{4}$. 0 presente estudo investigou essa relação apenas em idosos, evidenciando que esta relação se mantém para este público. Portanto, esses resultados somam-se à pesquisas já existentes que atesta que a solidão está positivamente associada a maior risco para o desenvolvimento de depressão em idosos ${ }^{27}$.

Os resultados também demonstraram associações significativas entre solidão e BES, de forma que idosos que experimentam menos solidão apresentam melhores índices de BES, uma vez que apresentam mais chances de experimentar menos afetos negativos e maior satisfação com a vida e afetos positivos. Relações entre BES e solidão (aferida pela versão portuguesa curta da UCLA) também foram testadas em idosos portugueses ${ }^{28}$ e corroboram os resultados aqui apresentados, no sentido de que o BES parece constituir um fator de proteção ao desenvolvimento de solidão em idosos.

A AS também apareceu associada à solidão em idosos. A AS é uma medida amplamente utilizada como importante indicador de bem-estar de idosos, por tratar-se de uma medida recomendada pela Organização Mundial de Saúde para verificar a saúde das populações. Desta forma, diante de dados que atestam que a solidão apresenta relação direta tanto com aspectos da saúde mental quanto da saúde física das pessoas ${ }^{29}$, era esperado que a AS também estivesse associada à solidão em idosos, sugerindo que quanto pior a avaliação que o idoso faz da sua saúde, maior o risco para vivenciar solidão.

A investigação aqui apresentada também mostrou associações entre solidão e PAP, evidenciando que esses construtos estão de forma geral relacionados, porém em magnitudes distintas, quando se considera o tipo específico de atividade prazerosa. As relações de maior magnitude entre solidão e PAP foram observadas para as atividades sociais e de competência, seguida das atividades contemplativas.

A solidão apresentou relações mais fracas e de menor nível de significância com as atividades práticas e intelectuais. A forma de contato social que cada tipo de atividade prazerosa pressupõe, pode ajudar a explicar essas relações observadas. Por exemplo, nenhum tipo de contato social é necessário para que as atividades intelectuais sejam praticadas ${ }^{18}$, e desta forma, esse tipo de atividade apresentaria pouca relação com a solidão, uma vez que esta é um construto definido a partir da percepção subjetiva do indivíduo sobre suas relações sociais ${ }^{1}$.

As atividades sociais e de competência, por sua vez, foi o tipo de atividade que apresentou relação de maior magnitude com a solidão. Atividades desta natureza pressupõem contatos sociais gratificantes para quem as pratica, considerando que essas atividades descrevem 
situações em que o idoso geralmente expressa um senso de competência e utilidade na relação com outras pessoas ${ }^{18}$, o que pode refletir em avaliações mais positivas sobre os relacionamentos sociais e consequente atenuação de vivências de solidão.

No entanto, as atividades contemplativas também apresentaram uma maior magnitude de associação com solidão, e tais atividades não requerem o contato com outras pessoas para que ocorram, ao passo que as atividades práticas que englobam atividades desenvolvidas no contexto doméstico e comunitário e que por isso demandariam algum contato social ${ }^{18}$, foram as que menos se associaram à solidão. Esse dado pode ser explicado a partir da compreensão sobre a diferença entre solidão e solitude.

A solitude, em contraste com a definição de solidão, expressa um estado positivo em que o indivíduo deriva benefícios em estar só ${ }^{30}$, podendo este ser o caso das pessoas que praticam as atividades contemplativas, que consistem em eventos introspectivos nos quais o indivíduo está só, mas tem a oportunidade de expressar sentimentos positivos ${ }^{18} \mathrm{e}$, consequentemente, apresentar menos chances de vivenciar solidão. Um estudo brasileiro qualitativo com idosas revelou que essas mulheres relataram avaliar a solidão também como uma experiência positiva, que neste caso poderia ser considerada como solitude, pois de acordo com a percepção dessas idosas estar só favorece a prática de atividades prazerosas sem a presença de outras pessoas, servindo como condição para uma conexão com si próprio e uma vida agradável9 .

As atividades práticas estão menos relacionadas à solidão, provavelmente porque o fato de o idoso ter contatos com pessoas no lar ou mesmo na comunidade ao se envolve em atividades domésticas ou comunitárias, não necessariamente implica na experiência de relacionamentos satisfatórios e gratificantes que os protejam da solidão, e, por isso, haveria pouca relação entre esses construtos. Desta forma, a disponibilidade de contatos sociais é uma condição necessária para que o indivíduo derive suporte social e esteja menos vulnerável a experimentar solidão, mas não é garantia para tal.

0 estudo aqui demonstrado avança no sentido de fazer análise da frequência da solidão em amostra de idosos brasileiros, além de apontar quais tipos de atividades prazerosas, em específico, parecem proteger mais os idosos da solidão.

\section{CONCLUSÃo}

Os resultados deste estudo evidenciaram que a maioria dos idosos apresentou baixos níveis de solidão, bem como foram identificados prováveis fatores de risco para a solidão em idosos, sendo o caso da depressão e de uma pior AS. Ainda, a partir das relações observadas entre solidão com PAP e BES, constatou-se que idosos que experimentam maiores níveis de BES e praticam atividades prazerosas, sobretudo sociais/de competência e atividades contemplativas, estão mais protegidos de vivências de solidão.

Entretanto, o estudo apresentou como limitação contar com amostra de conveniência, e por isso não representativa da população idosa brasileira, sendo necessário ter cautela para realizar generalizações. Estudos futuros devem ocupar-se de investigar fatores de risco e de proteção para solidão em idosos a partir de amostragem mais representativas. Ainda, as relações observadas devem ser exploradas em mais detalhes em investigações futuras, como a partir do desenvolvimento de modelos de regressão e de equações estruturais para melhor elucidar a natureza das relações entre esses construtos.

As implicações dos resultados deste estudo são relevantes para o planejamento e implementação de intervenções que busquem prevenir a solidão em idosos, compreendida hoje como um problema de saúde pública. Desta forma, proporcionar oportunidades para que idosos busquem vivenciar maiores níveis de BES e realizar atividades que lhe sejam prazerosas, constituem importantes estratégias para a prevenção de vivências de solidão e consequente promoção da saúde mental. 


\section{REFERÊNCIAS}

1. Utz RL, Swenson KL, Caserta M, Lund D, DeVries B. Feeling lonely versus being alone: loneliness and social support among recently bereaved persons. J Gerontol Ser B, Psychol Sci Soc Sci. [Internet]. 2014 [citado em 30 out 2020]; 69(1):85-94. DOI: https://doi.org/10.1093/geronb/gbt075

2. Barroso SM, Baptista MN, Zanon C. Solidão como variável preditora na depressão em adultos. Estud Interdiscip Psicol. [Internet]. 2018 [citado em 21 out 2020]; 9(Supl 3):26-37. DOI: http://dx.doi.org/10.5433/2236-6407.2018v9n3suplp26

3. Barroso SM, Andrade VS, Midgett AH, Carvalho RGN. Evidências de validade da Escala Brasileira de Solidão UCLA. J Bras Psiquiatr. [Internet]. 2016 [citado em 21 out 2020]; 65(1):6875. DOI: http://dx.doi.org/10.1590/0047-2085000000105

4. Holt-Lunstad J. The potential public health relevance of social isolation and loneliness: prevalence, epidemiology, and risk factors. Public Policy Aging Rep. [Internet]. 2017 [citado em 30 out 2020]; 27(4):127-30. DOI: https://doi.org/10.1093/ppar/prx030

5. Write-St Clair VA, Neville S, Forsyth V, White L, Napier S. Indigenous ageing research feature: integrative review of older adult loneliness and social isolation in Aotearoa/New Zealand. Austr J Ageing [Internet]. 2017 [citado em 30 out 2020]; 36(2):114-23. DOI: https://doi.org/10.1111/ajag.12379

6. Azeredo ZAS, Afonso MAN. Solidão na perspectiva do idoso. Rev Bras Geriatr Gerontol. [Internet]. 2016 [citado em 21 out 2020]; 19(2):313-24. DOI: http://dx.doi.org/10.1590/1809-98232016019.150085

7. Monteiro SFS. Mudanças relacionadas com a idade e bem-estar subjetivo: a influência da saúde e da solidão. [dissertação]. Braga, Portugal: Universidade do Minho; 2019. 34p.

8. Cachioni M, Delfino LL, Yassuda MS, Batistoni SST, Melo RC, Domingues MARC. Bem-estar subjetivo e psicológico de idosos participantes de uma Universidade Aberta à Terceira Idade. Rev Bras Geriatr Gerontol. [Internet]. 2017 [citado em 21 out 2020]; 20(3):340-51. DOI: http://dx.doi.org/10.1590/1981-22562017020.160179

9. Carmona CF, Couto VVD, Scorsolini-Comin F. A experiência de solidão e a rede de apoio social de idosas. Psicol Estud. [Internet]. 2014 [citado em 30 out 2020]; 19(4):681-91. DOI: http://dx.doi.org/10.1590/1413-73722395510

10. Casemiro NV, Ferreira HG. Indicadores de saúde mental em idosos frequentadores de grupos de convivência. Rev SPAGESP [Internet]. 2020 [citado em 21 out 2020]; 21(2):83-96. Disponível em: http://pepsic.bvsalud.org/pdf/rspagesp/v21n2/v21n2a07.pdf

11. Instituto Brasileiro de Geografia e Estatística. Idosos indicam caminhos para uma melhor idade [Internet]. Rio de Janeiro: IBGE; 2020. [citado em 21 out 2020]. Disponível em: https://censo2021.ibge.gov.br/2012-agencia-de-noticias/noticias/24036-idosos-indicamcaminhos-para-uma-melhor-idade.html

12. Brucki SMD, Nitrini R, Caramelli P, Bertolucci PHF, Okamoto IH. Sugestões para o uso do mini-exame do estado mental no Brasil. Arq Neuro-Psiquiatr. [Internet]. 2003 [citado em 21 out 2020]; 61(3B):777-81. DOI: https://doi.org/10.1590/S0004-282X2003000500014

13. Russell D, Peplau LA, Cutrona CE. The revised UCLA Loneliness Scale: concurrent and discriminant validity evidence. J Pers Soc Psychol. [Internet]. 1980 [citado em 21 out 2020]; 39(3):472-80. DOI: https://doi.org/10.1037/0022-3514.39.3.472

14. Russell DW. UCLA Loneliness Scale (Version 3): reliability, validity, and factor structure. J Pers Assess. [Internet]. 1996 [citado 21 out 2020]; 66(1):20-40. DOI: https://doi.org/10.1207/s15327752jpa6601_2

15. Barroso SM, Andrade VS, Oliveira NR. Escala Brasileira de Solidão: análises de resposta ao item e definição dos pontos de corte. J Bras Psiquiatr. [Internet]. 2016 [citado 21 out 2020]; 65(1):76-81. DOI: http://dx.doi.org/10.1590/0047-2085000000106 
16. Rider KL, Thompson LW, Gallagher-Thompson D. California Older Persons Pleasant Events Scale: a tool to help older adults increase positive experiences. Clin Gerontol. [Internet]. 2016 [citado em 21 out 2020]; 39(1):64-83. DOI: https://doi.org/10.1080/07317115.2015.1101635 17. Ferreira HG, Barham EJ. Adaptação transcultural de um instrumento para avaliar o envolvimento de idosos em atividades prazerosas. Cad Saúde Pública [Internet]. 2013 [citado em 30 out 2020]; 29(12):2554-60. DOI: https://doi.org/10.1590/0102-311X00130212

18. Ferreira HG, Barham EJ. Estrutura Fatorial da Versão Brasileira do California Older Person's Pleasant Events Schedule. Aval Psicol. [Internet]. 2017 [citado 21 out 2020]; 16(4):405-14. DOI: http://dx.doi.org/10.15689/ap.2017.1604.12627

19. Diener E, Emmons RA. The independence of positive and negative affect. J Pers Soc Psychol. [Internet]. 1984 [citado em 21 out 2020]; 47(5):1105-17. DOI: https://doi.org/10.1037/00223514.47.5.1105

20. Diener E, Emmons RA, Larsen RJ, Griffin S. The Satisfaction With Life Scale. J Pers Assess. [Internet]. 1985 [citado em 21 out. 2020]; 49(1):71-5. DOI: https://doi.org/10.1207/s15327752jpa4901_13

21. Albuquerque AS, Tróccoli BT. Desenvolvimento de uma escala de bem-estar subjetivo. Psicol Teor Pesqui. [Internet]. 2004 [citado em 21 out. 2020]; 20(2):153-64. DOI: http://dx.doi.org/10.1590/S0102-37722004000200008

22. Albuquerque FJB, Sousa FM, Martins CR. Validação das escalas de satisfação com a vida e afetos para idosos rurais. Psico [Internet]. 2010 [citado em 21 out. 2020]; 41(1):85-92. Disponível em: https://revistaseletronicas.pucrs.br/index.php/revistapsico/article/view/5110/5220

23. Sheikh JI, Yesavage JA. Geriatric Depression Scale (GDS): recent evidence and development of a shorter version. Clin Gerontol. [Internet]. 1986 [citado em 21 out 2020]; 5(1-2):165-73. DOI: https://doi.org/10.1300/J018v05n01_09

24. Almeida OP, Almeida SA. Confiabilidade da versão brasileira da Escala de Depressão em Geriatria (GDS) versão reduzida. Arq Neuro-Psiquiatr. [Internet]. 1999 [citado em 21 out. 2020]; 57(2B):421-6. DOI: https://doi.org/10.1590/S0004-282X1999000300013

25. Wang G, Zhang X, Wang K, Li Y, Shen Q, Ge X, et al. Loneliness among the rural older people in Anhui, China: prevalence and associated factors. Int J Geriatr Psychiatry [Internet]. 2011 [citado em 30 out 2020]; 26(11):1162-8. DOI: https://doi.org/10.1002/gps.2656

26. Anil R, Prasad KN, Puttaswamy M. The prevalence of loneliness and its determinants among geriatric population in Bengaluru City, Karnataka, India. Int J Community Med Public Health

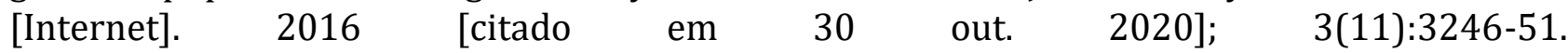
DOI: http://dx.doi.org/10.18203/2394-6040.ijcmph20163944

27. Oliveira LM, Abrantes GG, Ribeiro GS, Cunha NM, Pontes MLF, Vasconcelos SC. Solidão na senescência e sua relação com sintomas depressivos: revisão integrativa. Rev Bras Geriatr Gerontol. [Internet]. 2019 [citado em 21 out 2020]; 22(6):e190241. DOI: https://doi.org/10.1590/1981-22562019022.190241

28. Neto F. Psychometric analysis of the short-form UCLA Loneliness Scale (ULS-6) in older adults. Eur J Ageing [Internet]. 2014 [citado em 30 out 2020]; 11(4):313-9. DOI: https://doi.org/10.1007/s10433-014-0312-1

29. Richard A, Rohrmann S, Vandeleur CL, Schmid M, Barth J, Eichholzer M. Loneliness is adversely associated with physical and mental health and lifestyle factors: results from a Swiss national survey. Plos One [Internet]. 2017 [citado em 30 out 2020]; 12(7):1-18. DOI: https://doi.org/10.1371/journal.pone.0181442

30. Long CR, Averill JR. Solitude: an exploration benefits of being alone. J Theory Soc Behav. [Internet]. 2003 [citado em 30 out 2020]; 33(1):21-44. DOI: https://doi.org/10.1111/14685914.00204 


\section{CONTRIBUIÇÕES}

Heloísa Gonçalves Ferreira foi responsável pela pesquisa, análise de dados e revisão. Níldila Villa Casemiro contribuiu com a coleta de dados e redação.

\section{Como citar este artigo (Vancouver)}

Ferreira HG, Casemiro NV. Solidão em idosos e fatores associados. REFACS [Internet]. 2021 [citado em inserir dia, mês e ano de acesso]; 9(1):90-98. Disponível em: inserir link de acesso. DOI: inserir link do DOI

\section{Como citar este artigo (ABNT)}

FERREIRA, H. G.; CASEMIRO, N. V. Solidão em idosos e fatores associados. REFACS, Uberaba, MG, v. 9, n. 1, p. 90-98, 2021. DOI: inserir link do DOI. Disponível em: inserir link de acesso. Acesso em: inserir dia, mês e ano de acesso.

\section{Como citar este artigo (APA)}

Ferreira, H.G., \& Casemiro, N.V. (2021). Solidão em idosos e fatores associados. REFACS, 9(1), 90-98. Recuperado em inserir dia, mês e ano de acesso de inserir link de acesso. DOI: inserir link do DOI. 